\title{
Versorgungsforschung - evidenzbasiert Ein Kurzüberblick und Implikationen
}

\author{
Evidence-Based Health Services Research - A Short Review and Implications
}

\author{
Autoren \\ E. Gräßel ${ }^{1 *}$, C. Donath ${ }^{1 *}$, A. Hollederer ${ }^{2}$, H. Drexler ${ }^{3}$, J. Kornhuber ${ }^{4}$, A. Zobel ${ }^{5}$, P. Kolominsky-Rabas ${ }^{6}$ \\ Institute \\ Die Institutsangaben sind am Ende des Beitrags gelistet
}

\section{Schlüsselwörter \\ - Versorgungsforschung \\ - Evidenz \\ - Versorgungsoptimierung \\ - Review \\ - Kosten-Nutzen-Relation}

\author{
Key words \\ - health services research \\ - evidence \\ - care optimization \\ - review \\ - cost-utility analysis
}

\section{Bibliografie}

DOI http://dx.doi.org/

10.1055/s-0034-1382042

Online-Publikation: 23.9.2014

Gesundheitswesen 2015;

77: 193-199

(c) Georg Thieme Verlag KG Stuttgart · New York

ISSN 0941-3790

\section{Korrespondenzadresse}

Prof. Dr. med. Elmar Gräßel

Zentrum für Medizinische

Versorgungsforschung

Psychiatrische Universitätsklinik

Erlangen

Schwabachanlage 6

Erlangen 91054

elmar.graessel@uk-erlangen.de

\section{License terms}

\section{Zusammenfassung}

\section{$\nabla$}

Ausgangspunkt: Versorgungsforschung zielt darauf ab, Erkenntnisse darüber zu generieren, wie Prozesse der Versorgung von kranken Menschen, die medizinische Leistungen in Anspruch nehmen, ablaufen und welche Auswirkungen sie in der Anwendungsroutine auf die Beteiligten und auf das Gesundheitssystem haben.

Ausrichtung der theoretischen Arbeit: Im ersten Teil der Arbeit wird eine kompakte Übersicht über die aktuellen Definitionen und die Einordnung des Konzepts Versorgungsforschung gegeben. Im zweiten Teil wird dargestellt, wie evidenzbasierte Versorgungsforschung eingesetzt werden kann, um Verbesserungen im Gesundheitswesen zu erzielen. Das Konzept wird als „Kreislauf der Versorgungsoptimierung“ bezeichnet. Im ersten Schritt wird die bestehende Versorgungssituation hinsichtlich ihrer Defizite und der Gründe hierfür analysiert. Im zweiten Schritt wird ein bedeutsames Versorgungsziel definiert. Im dritten Schritt wird eine neue Vorgehensweise oder die Verbesserung einer vorhandenen Vorgehensweise konzipiert, um dieses Ziel zu erreichen. Im vierten Schritt wird eine vergleichende empirische Studie mit möglichst anspruchsvollem Studiendesign durchgeführt, um diese verbesserte Versorgungsstrategie im Vergleich zur bisher üblichen Vorgehensweise zu prüfen. Eine gesundheitsökonomische Evaluation ist dabei mit durchzuführen. Zeigen die Ergebnisse keine oder nur wenige Vorteile gegenüber der bisherigen Vorgehensweise, beginnt der „Kreislauf“ wieder mit Schritt 3. Sind die Ergebnisse jedoch signifikant, versorgungsrelevant und effizient - auch im Sinne gesundheitsökonomischer Überlegungen, werden im fünften Schritt Strategien der Implementierung ermittelt und erprobt. Gegebenenfalls werden in einem sechsten Schritt die Implementierungsfolgen

\section{Abstract \\ $\nabla$}

Background: Health services research aims to generate knowledge about care processes of people with illnesses who access health-care services. In addition, the consequences of those processes in the care routine concerning the involved persons and the health system are analyzed. Concept of the theoretical work: In the first part of the manuscript, an overview concerning the current definitions and subsumptions of the concept of health services research is given. The second part of the manuscript focuses on demonstrating how evidence-based health services research can be used to enable optimization of the care system. The concept is called the "circle of care optimization". In the first step the current care situation concerning its deficits and their reasons is analyzed. In the second step a relevant care goal is defined. In the third step an improvement of an existing care process is developed to achieve the defined care goal. In the fourth step, a comparative empirical study with a high-quality study design is carried out, to assess whether the improved care process is superior to the current care as usual. A health economic evaluation will be performed if applicable. If the results show no or only small advantages, the "circle" starts again with step 3. However, if the results show a significant effect in favour of the new care process and are relevant for the delivery of care and efficient in the context of health economics, a fifth step will be performed which involves developing and testing strategies for implementation. Where relevant, the consequences of implementation are investigated in a sixth step. A "best-practice" practical example is demonstrated to illustrate the "circle of care optimization". Conclusions: Conclusions are derived by illustrating future challenges for health services research.

*geteilte Erstautorenschaft 
erforscht. Diese Vorgehensweise wird anschließend anhand von einem „best-practice“-Beispiel veranschaulicht.

Schlussfolgerung: Ausgehend von den in dieser Arbeit vorgestellten Versorgungsforschungskonzepten werden im letzten Abschnitt Herausforderungen abgeleitet.

\section{Übersicht Versorgungsforschung (Teil I)}

$\nabla$

Definition, Einordnung, Abgrenzung, Aufgaben, Umsetzung

Definition

Die derzeitige klassische wissenschaftliche Definition von „health services research“ leitet sich aus einer Veröffentlichung der Academy for Health Services Research and Health Policy vom Juni $2000 \mathrm{ab}$, die (vereinfacht) Versorgungsforschung als Feld sieht, welches Zugang, Qualität und Kosten von gesundheitlicher Versorgung und deren Einfluss auf Gesundheit und Wohlbefinden untersucht: "the multidisciplinary field of scientific investigation that studies how social factors, financing systems, organizational structures and processes, health technologies, and personal behaviors, affekt access to health care, the quality and cost of health care, and ultimately our health and wellbeing. Its research domains are individuals, families, organizations, institutions, communities, and populations" [1].

Inzwischen gibt es weitere Vorschläge zur Optimierung der wissenschaftlichen Definition, die sich im Wesentlichen darauf beziehen, das Ziel von „Health Services Research“, also Versorgungsforschung, in die Definition mit aufzunehmen. Das heißt, klarzustellen, dass man Versorgungsforschung durchführt, um die Versorgung effektiver, gerechter und effizienter zu gestalten: „attempts to improve the effectiveness, equity, or efficiency of healthcare" [2].

Nach der Definition des Deutschen Netzwerks Versorgungsforschung (DNVF) wird Versorgungsforschung als „ein multidisziplinärer Ansatz zur Erforschung der Umsetzung wissenschaftlicher Erkenntnisse in die Praxis der Gesundheitsversorgung hinsichtlich ihrer Wirkung auf Qualität und Effizienz in individueller und sozioökonomischer Perspektive“ gesehen [3]. Nach einem aktuellen Lehrbuch der Versorgungsforschung „hat sich in Deutschland folgende Definition durchgesetzt“: „Versorgungsforschung ist ein fachübergreifendes Forschungsgebiet, das die Kranken- und Gesundheitsversorgung und ihre Rahmenbedingungen beschreibt und kausal erklärt, zur Entwicklung wissenschaftlich fundierter Versorgungskonzepte beiträgt, die Umsetzung neuer Versorgungskonzepte begleitend erforscht und die Wirksamkeit von Versorgungsstrukturen und -prozessen unter Alltagsbedingungen evaluiert" [4]. Alle bisherigen Konzepte der Versorgungsforschung beruhen laut Pfaff \& Schrappe [4] auf den 3 Grundkonzepten Ergebnisorientierung, Multidisziplinarität/Multiprofessionalität sowie Patientenorientierung.

Die Bundesärztekammer (BÄK) [5] orientiert sich an diesen Definitionen und stellt darüber hinaus die Alltagsorientierung in den Vordergrund als eine „grundlagen- und problemorientierte fachübergreifende Forschung“. So ist Versorgungsforschung „die wissenschaftliche Untersuchung der Versorgung von Einzelnen und der Bevölkerung mit gesundheitsrelevanten Produkten und Dienstleistungen unter Alltagsbedingungen. Sie studiert, wie Finanzierungssysteme, soziale und individuelle Faktoren, Organisationsstrukturen und -prozesse und Gesundheitstechnologien den Zugang zur Kranken- und Gesundheitsversorgung sowie deren Qualität und Kosten letztendlich unsere Gesundheit und unser Wohlbefinden beeinflussen“.

Der Begriff Bevölkerung schließt auch gesunde Klienten mit ein, die bspw. im Rahmen von Früherkennungsmaßnahmen (fälschlicherweise meist als Vorsorgeuntersuchung bezeichnet) aus unterschiedlichen Anlässen (pädiatrische Untersuchungen, Untersuchung nach dem Jugendarbeitsschutzgesetz, arbeitsmedizinische Vorsorge, Krebsfrüherkennungsprogramme u.a.) Leistungen in Anspruch nehmen.

Insbesondere thematisiert Versorgungsforschung die in vielen Gesundheitssystemen nachweisbare Kluft zwischen den aktuellen wissenschaftlichen Erkenntnissen und der realen Versorgungssituation [6].

Die Definition von Versorgungsforschung der BÄK [7] enthält 5 entscheidende Punkte (Inhalte teils gekürzt):

1. Beschreibung der Einflüsse, Prozesse und Ergebnisse der Patientenversorgung mit wissenschaftlichen Methoden

2. Erklärung von kausalen Bedingungszusammenhängen soweit möglich

3. Neuentwicklung theoretisch fundierter und empirisch geprüfter Versorgungskonzepte

4. Erforschung der Umsetzung neu entwickelter Versorgungskonzepte (begleitend oder folgend)

5. Evaluation von Versorgungsstrukturen, -prozessen, -konzepten unter Alltagsbedingungen

Die Punkte 1 und 2 sind nach Pfaff [8] als Grundlagenforschung in der Versorgungsforschung zu verstehen, die Punkte 3-5 hingegen als anwendungsorientierte Versorgungsforschung.

Laut BÄK [7] soll das Ziel von Versorgungsforschung auch sein, Optimierungsprozesse zu fördern und Risiken zu mindern.

\section{Einordnung}

Gegenstand von Versorgungsforschung ist das Versorgungssystem. Das Versorgungssystem besteht aus einer Anzahl von Elementen, die jedes für sich klar umrissen sind, aber untereinander in Beziehung stehen. Diese Beziehungen sind nicht monokausal und unidirektional, sondern die Elemente stehen miteinander in Wechselwirkung. Daraus ergibt sich eine gewisse Komplexität, die sich dann auf die Verstehbarkeit und die Beeinflussbarkeit des Systems auswirkt [8].

Zur weiteren theoretischen Fundierung wird von den meisten Autoren das systemtheoretische Modell des Versorgungssystems genutzt. Mittels dieses Modells lassen sich die einzelnen Forschungsinhalte der Versorgungsforschung klassifizieren (๑ Abb. 1: adaptiert nach Pfaff [8]).

Man geht davon aus, dass das Versorgungssystem nicht nur sehr komplex ist, sondern letztlich auch nicht klar ist, wie genau die einzelnen Einflüsse und Mittel, die in das System fließen, das Ergebnis determinieren (Stichwort „Black Box“). Aus diesem Grund versucht man die Forschung in verschiedene Abschnitte aufzugliedern. Einerseits wird untersucht, welche von außen einwirkenden Faktoren das System beeinflussen (Input). Zum anderen werden die Leistungen, die in und durch das System erbracht werden (Output) betrachtet. Und letztlich wird das Versorgungsergebnis (Outcome), auch als Kosten-Nutzen-Relation analysiert. Um die Komplexität des Versorgungssystems besser zu verstehen, beschreibt die „Throughput“-Perspektive dagegen, welche Strukturen und Prozesse im Versorgungssystem wirken, welche Technologien eingesetzt werden, damit letztlich aus einem bestimmten Input ein bestimmter Output und ein Outcome erzielt wird. 


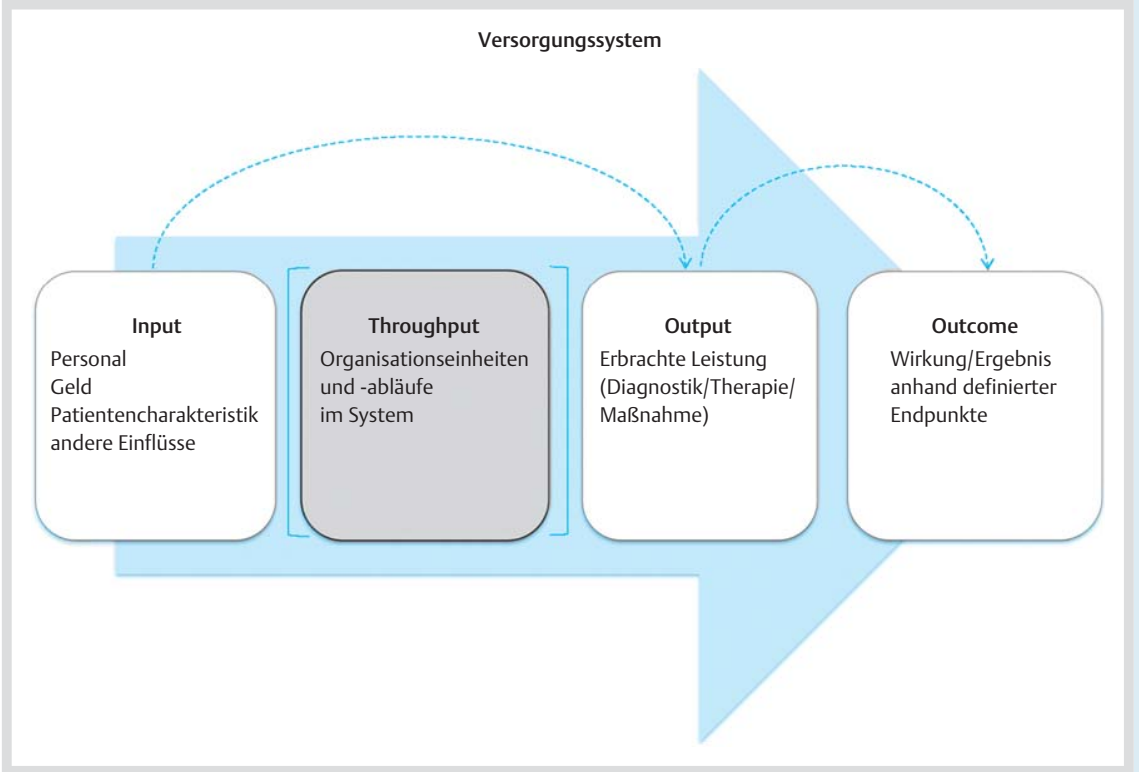

Abb. 1 Systemtheoretisches Modell des Versorgungssystems (adaptiert nach Pfaff [8]).
Aus unserer Perspektive scheint der derzeitige Stand der Versorgungsforschung so zu sein, dass meistens nicht alle Perspektiven des Versorgungsgeschehens einer Krankheit gleichzeitig ins Visier genommen werden. Der Fokus liegt oft auf der beschreibenden Perspektive einzelner Systemelemente (siehe folgende Abschnitte) oder aber auf einer unidirektionalen Effizienzanalyse (Input vs. Output).

Unter Input-Forschung versteht man nicht nur die Analyse der zugeführten Ressourcen (Personal, Material, Geld), sondern auch der Faktoren, die den Eintritt in das Versorgungssystem beeinflussen (Patientencharakteristiken wie: Bedarf, Inanspruchnahme, usw.) bzw. behindern (Barrieren) sowie Versorgungsvoraussetzungen [8]. Hier spielen auch die unterschiedlichen Rahmenbedingungen der gesetzlichen und privaten Krankenversicherung eine wesentliche Rolle.

Die Throughput-Forschung analysiert Versorgungsstrukturen und -prozesse des Versorgungssystems aus organisatorischer, diagnostischer und therapeutischer Perspektive. Sie untersucht die Kombination aus Gesundheitsleistung und Kontext in Form von Versorgungsstrukturen und -prozessen. Systematische Bewertungen von versorgungsrelevanten Gesundheitstechnologien (z.B. Arzneimittel, Medizinprodukte) im Sinne von Health Technology Assessment (HTA) werden aktuell unter dieser Perspektive beleuchtet [9]. Versorgungstechnologien und deren Anwendungsvoraussetzungen und -möglichkeiten sind hier von großer Bedeutung.

Die Output-Forschung betrachtet die unmittelbar erbrachten Versorgungsleistungen. Diese Betrachtung erfolgt zunächst unabhängig vom Versorgungsergebnis, also der Wirkung der Versorgungsleistung. Von besonderem Interesse sind Menge und Qualität der Versorgungsleistung, die am Bedarf und der maximal möglichen Qualität relativiert werden müssen. Hier zeigt sich einerseits, dass die objektive Bedarfsermittlung bisher in Forschung und Wissenschaft zu wenig Beachtung fand. Zum anderen ist die Versorgungsqualität erst in jüngerer Zeit Gegenstand der Wissenschaft.

Die Outcome-Forschung evaluiert das Erreichen der beabsichtigten Wirkung durch die Versorgungsleistung. Das heißt z.B. der Gewinn oder Verlust an Gesundheit, Lebensqualität, Selbständigkeit, Wohlbefinden, Arbeitsfähigkeit usw. Outcome Research ist nach den US-Autoren Clancy \& Eisenberg „the study of the end results of health services that takes patients` experiences, preferences, and values into account" [10]. Neben patientenrelevanten Endpunkten wie Mortalität und Morbidität, die vor allem auf die Lebensdauer abheben, werden zunehmend Parameter betrachtet, die Aktivitäten und Teilhabeaspekte wie Kommunikation, Mobilität, Selbstversorgung, Teilnahmemöglichkeit am soziales Leben usw. abbilden. Die bisherige Betrachtung des kranken Menschen mit dem Fokus auf seine Defizite wird zunehmend abgelöst von einer Betrachtungsweise, welche die erhaltenen und förderungsfähigen Fähigkeiten in den Mittelpunkt stellt (z.B. International Classification of Functioning, Disability and Health [11]), Dieses Prinzip kommt vor allem bereits in der Sozialmedizin zur Anwendung.

\section{Abgrenzung}

Versorgungsforschung unterscheidet sich von klinischer Forschung in erster Linie dadurch, dass klinische Forschung auf Wirksamkeitsnachweise von therapeutischen Interventionen abzielt, die oftmals unter Idealbedingungen, die nicht den realen Alltag der Betroffenen abbilden, erbracht werden (efficacy) (nach http://www.cochrane.de/de/cochrane-glossar). Bei Versorgungsforschung im Zusammenhang mit therapeutischen Interventionen geht es im nachfolgenden Schritt darum, diese Interventionen (=Versorgungsleistungen), die sich im klinischen Forschungssetting als wirksam erwiesen haben, auf ihre Wirksamkeit im Alltag des realen Versorgungssystems (mit seinen multiplen Einflüssen und Wechselwirkungen) zu untersuchen (effectiveness).

Versorgungsforschung orientiert sich zum einen auf bereits vorhandene Versorgungsstrukturen und -prozesse und zum anderen auf innovative, evidenzbasierte Weiterentwicklungen unter Berücksichtigung und Erhalt bestehender Strukturen. Versorgungsforschung hat gegenüber klinischer Forschung auch das Ziel beratend und regulierend zu Versorgungssystementscheidungen beizutragen.

Die Versorgungsforschung analysiert die Alltagswirksamkeit der Methoden und Maßnahmen in der gesundheitsrelevanten Versorgung von gesunden und kranken Menschen unter realen Bedingungen und kontrolliert im Gegensatz zur klinischen Forschung die moderierenden Faktoren. Das Forschungsinteresse liegt vor allem auf der Ergebnisqualität, die durch die Struktur- 


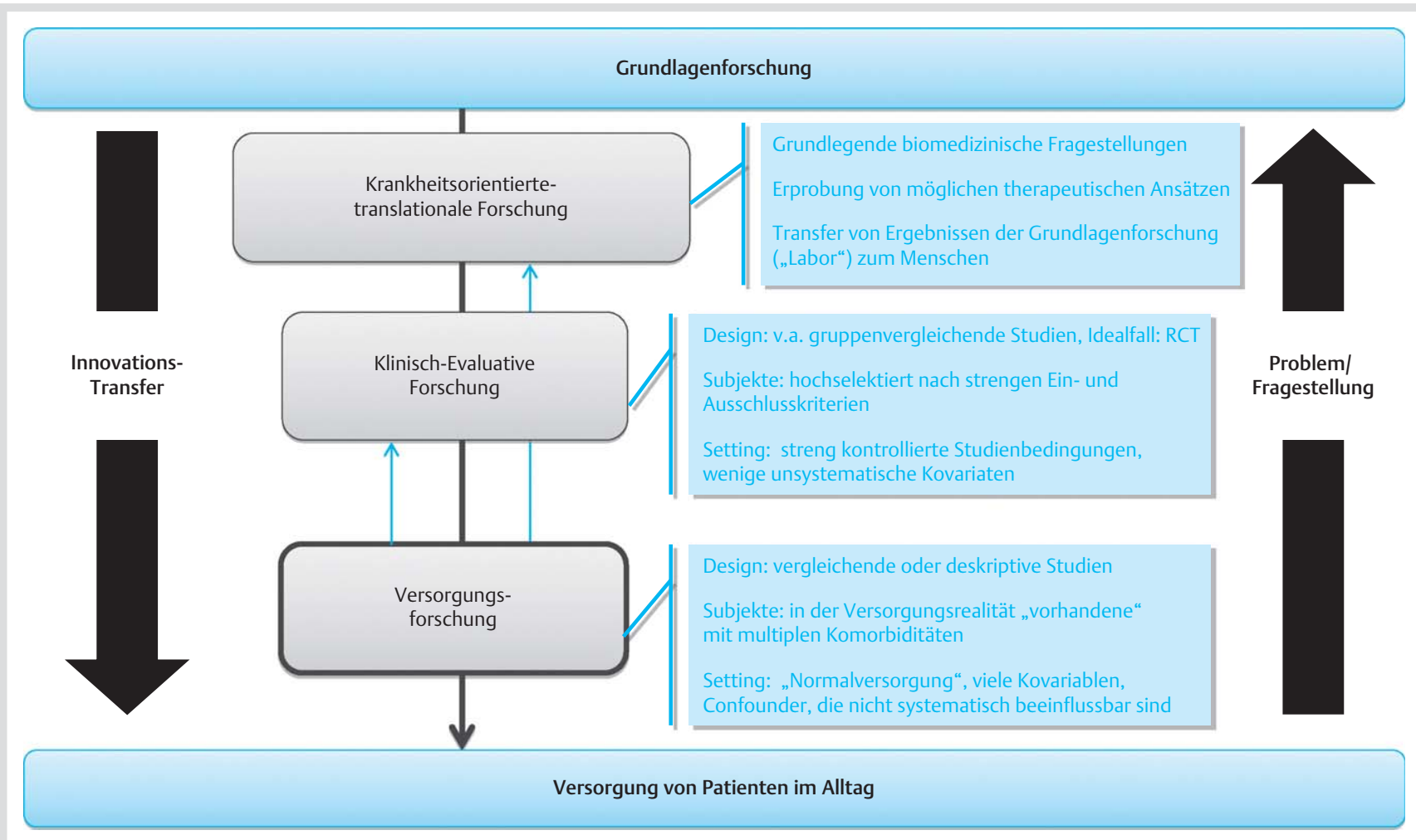

Abb. 2 Positionierung der Versorgungsforschung in der medizinischen Forschung (adaptiert nach Neugebauer [31]).

und Prozessqualität im Versorgungsgeschehen maßgeblich beeinflusst werden kann.

In $\bullet$ Abb. 2 ist ersichtlich, dass im Forschungsablauf einer evidenzbasierten Medizin die Versorgungsforschung idealerweise routinemäßig einbezogen wird, bevor eine weitreichende flächenmäßige Implementation einer Versorgungsleistung erfolgt. Versorgungsforschung kann aber auch Ausgangspunkt z.B. bei der Entwicklung neuer Versorgungskonzepte fungieren. Versorgungsforschung ist aber nicht nur der letzte Schritt „Forschung“ vor der Praxiseinführung. Versorgungsforschung kann auch Defizite und Bedarf aufzeigen und bspw. zu klinischer Forschung anregen - nämlich dann wenn offensichtlich wird, dass die Versorgung mit den Standardtherapien zu unbefriedigenden Ergebnissen im Versorgungsalltag führt. Damit ist Versorgungsforschung nicht nur unidirektional, sondern hat auch Rückkopplungsmöglichkeiten. Leider wird sie derzeit viel zu selten vor der weitreichenden flächenmäßigen Implementierung einer Versorgungsleistung einbezogen und damit die Chance vertan, evidenzbasierte Entscheidungen zu treffen.

\section{Aufgaben}

Neben den in der methodischen Definition der BÄK genannten 5 Punkten, die die primären Aufgaben der Versorgungsforschung klar umreißen, kristallisieren sich weitere Kernaufgaben heraus [12]. 3 davon sollen hier beispielhaft genannt werden:

\section{Definition von Unterversorgung und Überversorgung so- wie Fehlversorgung}

Die Feststellung von Unter- oder Überversorgung ist nicht trivial, weil sie sich nicht auf die bloße Darstellung von Häufigkeiten in der Anwendung oder Nichtanwendung bestimmter Maßnahmen reduzieren lässt. Vielmehr müssen für die Interpretation solcher meist regionaler oder sozial bedingter Unterschiede in der Versorgung eine Vielzahl von konfundie- renden Variablen, d.h. erklärenden Fakten, herangezogen werden. Dies können bestimmte Häufungen von Patientencharakteristika sein, Besonderheiten in den Versorgungsstrukturen vor Ort, idealerweise auch die Wunschvorstellung hinsichtlich Behandlungsergebnis des einzelnen Patienten (der für ihn persönlich relevante Endpunkt) und andere im Versorgungssystem wirkende Einflüsse. Diese Faktoren in die Interpretation von Versorgungsunterschieden zu integrieren ist eine wesentliche Aufgabe der Versorgungsforschung.

2. Erforschung von patientenrelevanten Endpunkten zur Spezifizierung der Outcomeforschung

Die Feststellung, ob eine Versorgungsleistung (ein Output) wirklich erfolgreich war, wird bisher mit der Outcomeanalyse erforscht. Das Ergebnis hängt dabei entscheidend davon ab, welches Outcome gewählt wurde. Bisher war es nicht selten, dass sogenannte „Surrogate“ als Ergebnisvariable betrachtet wurden. Dieses sind Ersatzparameter, die quasi eine Vorstufe eines klinischen Parameters darstellen. Für die korrekte Verwendung muss das Surrogat validiert sein und ein kausaler Zusammenhang zwischen Surrogat und klinischem Parameter nachgewiesen werden (therapeutischer Effekt auf Surrogat $\rightarrow$ kausaler Effekt auf Zielvariable). Diese Validierung ist jedoch bis heute für viele verwendete Surrogate nicht durchgeführt, sodass es schon zu medizinisch falschen Schlüssen kam [13]. Die Alternative wäre, patientenrelevante Endpunkte zu entwickeln, um ein Outcome zu beurteilen und infolge dessen Versorgungsentscheidungen anhand solcher validierter Endpunkte zu treffen. Dies bedeutet, die Patientenpräferenzen und den Patientennutzen bei Entscheidungsprozessen prioritär einzubeziehen [14]. Zu diesem Punkt erfolgt eine detaillierte Darstellung in diesem Heft von Apfelbacher und Loss. 
Tab. 1 Evidenzklassen (in Anlehnung an Deutsches Netzwerk Evidenzbasierte Medizin e.V., www.ebm-netzwerk.de).

\begin{tabular}{|c|c|}
\hline Evidenzklassen & Beschreibung \\
\hline la & $\begin{array}{l}\text { Evidenz aufgrund einer systematischen Übersichtsarbeit (Meta-Analyse) mit mindestens } 3 \text { randomisierten kontrollierten Studien } \\
\text { (randomized controlled trial, RCT) }\end{array}$ \\
\hline Ib & Evidenz aufgrund mindestens einer RCT (oder einer Meta-Analyse von weniger als 3 RCT) \\
\hline Ila & Evidenz aufgrund mindestens einer kontrollierten nicht-randomisierten Studie mit methodisch hochwertigem Design \\
\hline Illb & Evidenz aufgrund mindestens einer quasi-experimentellen Studie mit methodisch hochwertigem Design \\
\hline III & Evidenz aufgrund einer nicht-experimentellen deskriptiven Studie (Vergleichsstudie, Korrelationsstudie, Fallserie) \\
\hline IV & Evidenz aufgrund von Berichten/Empfehlungen von Expertenkomitees, klinische Erfahrung anerkannter Autoritäten \\
\hline
\end{tabular}

\section{Politikfolgeforschung}

Dies bedeutet im letzten Schritt, noch nach der Implementation von Versorgungsaspekten im deutschen Gesundheitssystem, zu überprüfen, welche Veränderungen (Outcome) dieses Gesetz gebracht hat (nachdem beschrieben wurde, wie das Gesetz von den einzelnen Beteiligten umgesetzt wurde). Als ein Beispiel könnte die Evaluation des Gesetzes zur Verbesserung der Versorgungsstrukturen in der gesetzlichen Krankenversicherung (GKV-VStG) genannt werden, welches zum 01.01.2012 in Kraft getreten ist [15].

Eine breit gefächerte Aufstellung von weiteren Aufgaben der Versorgungsforschung findet sich im Memorandum „Methoden für die Versorgungsforschung“ des Deutschen Netzwerks für Versorgungsforschung (DNVF) [16-18] und wird außerdem aktuell diskutiert im nationalen Aktionsplan für Versorgungsforschung des DNVF [19].

\section{Umsetzung}

Studien der Versorgungsforschung sollten sich bezüglich des methodischen Vorgehens und der Standards am „Memorandum III: Methoden für die Versorgungsforschung“ des Deutschen Netzwerks für Versorgungsforschung (DNVF) orientieren $[16,17]$.

\section{Evidenzbasierte Versorgungsforschung (Teil II)}

Wie in der vorausgehenden Übersicht dargestellt, wurde in der Versorgungsforschung bisher der Aspekt des Evidenzgrades von Erkenntnissen kaum Beachtung geschenkt, da viele der behandelten Fragestellungen hauptsächlich mit beschreibenden Methoden bearbeitet wurden.

Betrachten wir jedoch eine Maßnahme, die das Versorgungsgeschehen gezielt verändert, besteht die Möglichkeit, diese Veränderung im Vergleich zur bisherigen Vorgehensweise, eventuell zum bisher üblichen Standard zu untersuchen.

Damit kann das Konzept des gestaffelten Erkenntniswertes, bekannt aus der klinischen Therapieforschung als Evidenzgrad ( $\bullet$ Tab. 1), auch auf Fragen der Versorgungsforschung angewandt werden und der Grad des Erkenntniswertes erhöht werden; wir sprechen nun von „evidenzbasierter Versorgungsforschung“.

Voraussetzung für einen möglichst hohen Evidenzgrad ist die Verwendung einer möglichst elaborierten Forschungsmethodik, die das Studienergebnis beeinflussende Fehlerquellen minimiert. Vorgehensweisen, die aus experimentellen Studiendesigns stammen, sind hier hilfreich: (1) Verwendung von validen, reliablen und objektiven Instrumenten zur Operationalisierung des Ist-Zustands; (2) kontrollierte Vorgehensweise durch Definition der Vergleichssituation (3) Bildung von Hypothesen; (4) Festlegung der Rahmenbedingungen, unter denen die beiden Versorgungsoptionen untersucht werden sollen und (5) Berück- sichtigung von Outcome-Parametern mit möglichst hoher Validität und vor allem mit großer Versorgungsrelevanz.

Als nächstes wird im „Kreislauf der Versorgungsoptimierung“ theoretisch erläutert, wie eine Optimierung der Versorgung von kranken Menschen im Sinne der evidenzbasierten Versorgungsforschung erreicht werden kann ( $\bullet$ Abb. 3). Der „Kreislauf der Versorgungsoptimierung" besteht aus 6 Schritten:

Als erster Schritt wird eine Ausgangsanalyse vorgenommen, um bestehende Defizite in der aktuellen Versorgungssituation festzustellen.

Dies bildet die Grundlage für den zweiten Schritt, der darin besteht, ein konkretes Versorgungsziel festzulegen.

Im dritten Schritt wird eine neue Vorgehensweise, Maßnahme oder die Verbesserung eines vorhandenen Konzepts entwickelt, um dieses Ziel zu erreichen.

Im vierten Schritt wird eine vergleichende empirische Studie mit möglichst anspruchsvollem Studiendesign der Evidenzklasse Ib oder Ila durchgeführt, um die verbesserte Versorgungsstrategie im Vergleich zur bisher üblichen Vorgehensweise zu prüfen. Eine gesundheitsökonomische Evaluation ist dabei möglichst mit durchzuführen.

Zeigen die Ergebnisse keine oder nur wenige Vorteile gegenüber der bisherigen Vorgehensweise („-“ $\bullet$ Abb. 3), beginnt der „Kreislauf“ wieder mit Schritt 3. Sind die Ergebnisse jedoch signifikant, versorgungsrelevant und effizient ( „, “ in $\odot$ Abb. 3 ) auch im Sinne gesundheitsökonomischer Überlegungen, werden im fünften Schritt Strategien der Implementierung in die Regelversorgung oder zumindest in die „Breite“ der Versorgungslandschaft ermittelt und erprobt. Bei Implementationsschwierigkeiten sollten verbesserte Strategien entwickelt und erprobt werden.

Gegebenenfalls werden in einem sechsten Schritt die Folgen der Implementierung mit Versorgungsforschungsstudien erforscht.

Sollten weiterhin Versorgungsdefizite bestehen (Schritt 1), geht der „Kreislauf der Versorgungsoptimierung“ iterativ wieder mit Schritt 2, der Festlegung eines weiteren Versorgungsziels, weiter. Nachfolgend wird der „Kreislauf der Versorgungsoptimierung“ an einem Beispiel aus dem Bereich chronisch degenerativer Erkrankungen, hier der degenerativen Demenzen, veranschaulicht.

Der erste Schritt, die Ausgangsanalyse mündet in die Feststellung, dass bei der Versorgung von Menschen mit Demenz 3 Probleme vorherrschen: Umgang mit herausfordernden Verhaltensweisen [20], Nachlassen der alltagspraktischen Fähigkeiten gefolgt von einer zunehmenden Unselbstständigkeit im Alltag [21] sowie eine große, vor allem psychische Belastung der Pflegenden [22]. Bei näherer Betrachtung zeigte sich, dass es keine Versorgungsangebote, etwa Therapieansätze oder sonstige Maßnahmen, gibt, die einen praktisch relevanten Einfluss auf die alltagspraktischen Fähigkeiten ausüben. 


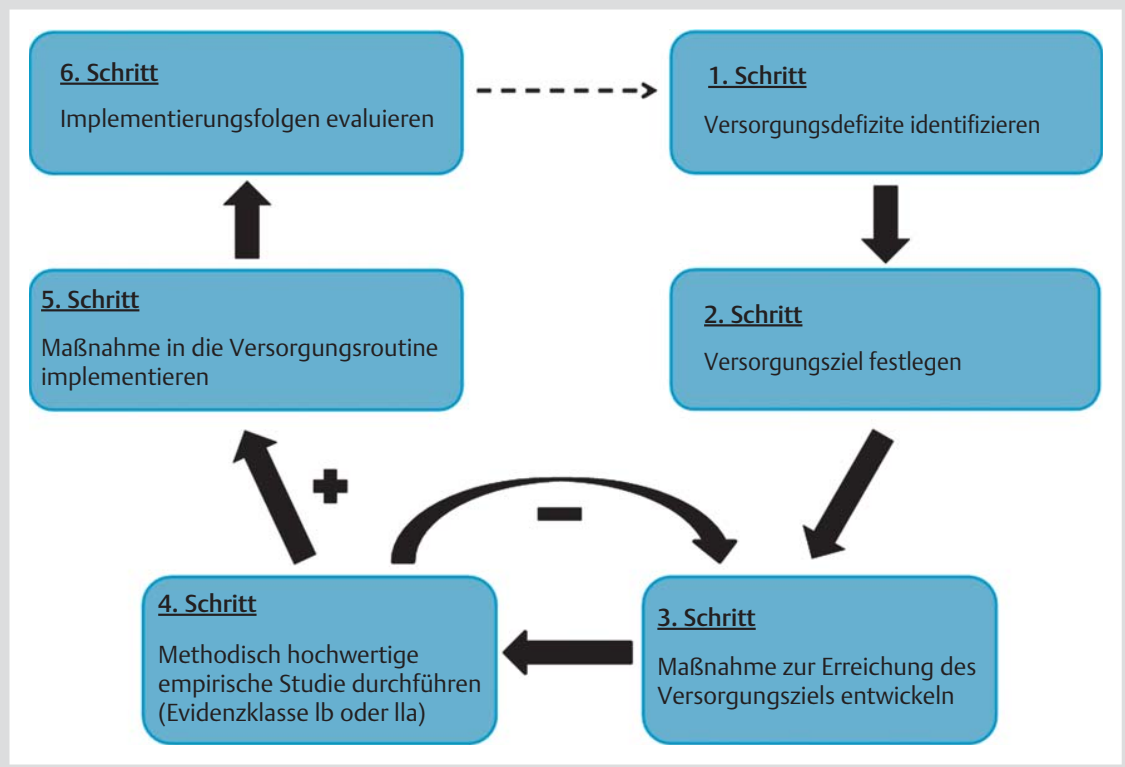

Abb. 3 Das Modell „Kreislauf der Versorgungsoptimierung“.

Deshalb wurde im zweiten Schritt als Versorgungsziel eine relevante Verlangsamung der Abnahme der alltagspraktischen Fähigkeiten festgelegt.

Unter Berücksichtigung der aktuellen wissenschaftlichen Erkenntnislage wurde im dritten Schritt angenommen, dass eine multimodale Intervention, bestehend aus kognitiven, psychomotorischen und alltagspraktischen Elementen, in der Lage sein könnte, die Progression des Nachlassens der alltagspraktischen Fähigkeiten in relevantem Ausmaß und für längere Zeit abzuschwächen. Dazu wurde eine standardisierte Umsetzung der Maßnahme, im Sinne eines Manuals entwickelt [23].

Zur Überprüfung der Wirksamkeit wurde im vierten Schritt eine randomisierte kontrollierte Studie durchgeführt, bei der die Kontrollbedingung die übliche Versorgung darstellte. Zur Erfassung der alltagspraktischen Fähigkeiten wurde ein Leistungstest verwendet $[24,25]$, der die einfach verblindete Messung der Zielvariable mit hoher Validität ermöglicht und aus versorgungsrelevanten Items besteht (z.B. Schleife binden können). Die erzielten Ergebnisse waren nicht nur signifikant, sondern zeichneten sich durch eine hohe Versorgungsrelevanz aus. So konnten die alltagspraktischen Fähigkeiten für mindestens 12 Monate durchgehend auf einem stabilen Niveau gehalten werden [26]. Zudem zeigten sich auch nach Beendigung der systematischen Intervention deutliche nachhaltige Effekte auf die alltagspraktischen Fähigkeiten der Betroffenen [27]. Außerdem konnte aufgezeigt werden, dass durch die Einsparung von Pflegezeit die multimodale MAKS-Intervention auch gesundheitsökonomisch interessant ist.

Als erste Strategie der Implementierung in die Versorgungsroutine wurde im fünften Schritt ein Schulungskonzept für die MAKSMaßnahme nach dem Prinzip „Train-the-Trainer“ entwickelt. Dies wurde und wird in Schulungen umgesetzt (www.maks-aktivie rungstherapie.de). Parallel dazu wurde das MAKS-Konzept mehreren Landespflegeausschüssen sowie Verbänden der gesetzlichen und privaten Kranken-/Pflegeversicherung vorgestellt.

Geplant ist, im sechsten Schritt zu ermitteln, welche Folgen die Einführung des MAKS-Konzepts in den betreffenden Einrichtungen für die von Demenz Betroffenen, aber auch für die dort tätigen Personen hat.

\section{Herausforderungen für die Versorgungsforschung} $\nabla$

Ausgehend von den in dieser Arbeit vorgestellten Versorgungsforschungskonzepten werden 4 Herausforderungen für die Versorgungsforschung und ihr Beitrag zur Optimierung der Versorgung der Zukunft abgeleitet:

1. Versorgungsforschung lässt sich theoretisch sehr gut in der Systemperspektive betrachten und den einzelnen Bestandteilen des Systemmodells zuordnen. Die wissenschaftlich-praktische Betrachtung des Versorgungsgeschehens in der umfassenden Systemperspektive scheint jedoch in der Praxis eher gering ausgeprägt zu sein. Trotz der Berechtigung von Teilbetrachtungen, die bspw. detailliert die Inanspruchnahme von Leistungen beschreiben (Input-Forschung), ist die Herausforderung anzunehmen, in neuen Studien die Gesamtsystemperspektive der Versorgung bspw. für bestimmte Personengruppen, Regionen oder Krankheitsentitäten einzunehmen.

2. Eine weitere Herausforderung in der Versorgungsforschung wird die Umsetzung eines Paradigmenwechsels von den von Experten festgelegten Zielpunkten hin zu von Patienten bestimmten Zielpunkten (Patient Reported Outcomes, „PRO“) sein [28]. In den USA wird diese Vorgehensweise bereits zur Bewertung von Medizintechnologien umgesetzt [29]. Detaillierte Ausführungen zu diesem Thema enthält außerdem die Arbeit von Apfelbacher und Loss in der gleichen Ausgabe.

3. Evidenzbasierte Versorgungsforschung setzt die konsequente Anwendung einer elaborierten Forschungsmethodik voraus. Dieser Aufwand verursacht relativ hohe Kosten. Ein wichtiger Grund für die bisher relativ geringe Anzahl von Versorgungsforschungsstudien mit dem Anspruch, Ergebnisse auf dem Evidenzniveau Ib oder IIa zu erzielen, ist deshalb in der Finanzierung zu sehen. Die Summe von 500000 Euro für eine kontrollierte Studie mit ausreichender Fallzahl ist ein realistischer Mindestbedarf. Im Unterschied zu „klinischen Studien“, bei denen Therapieformen im klassischen Sinn erforscht werden, ist das Maßnahmenspektrum, das bei „evidenzbasierten Versorgungsforschungsstudien“ untersucht wird, viel breiter gefasst - z.B. der Einsatz eines aufsuchenden Beratungsangebots bei häuslicher Pflege mit der Zielsetzung, den Übertritt ins Pflegeheim zu verzögern [30]. Im 
Gegensatz zur Arzneimittelforschung, die aufgrund der Gewinnmöglichkeiten durch Patente größtenteils privatwirtschaftlich finanziert wird, ist es verhältnismäßig schwierig, Sponsoren für anspruchsvolle Versorgungsforschungsstudien zu finden. Hier greift die Verantwortung öffentlicher „Geldgeber“, wie Bundes- und Länderministerien, Deutsche Forschungsgemeinschaft sowie gesetzliche und private Krankenund Pflegeversicherung. In Frage kommen auch Interessensverbände und Institutionen wie z.B. die Bundesärztekammer. Dabei sollte die Vergabe der Mittel notwendigerweise durch öffentliche Ausschreibung und transparente Zuschlagserteilung geregelt werden. Obwohl diese Finanzierungen gegebenenfalls Steuerzahler und/oder Versicherte belasten, sind sie gerechtfertigt, da durch eine „bessere“ Versorgung mittelfristig sowohl Patienten bzw. Versicherte als auch die gesamte Gesellschaft durch mehr Gesundheit bei gleichen oder geringeren Kosten profitieren.

4. Die Implementierung einer neuen, evidenzbasierten Maßnahme in die Versorgungsroutine von kranken Menschen bedeutet Veränderung. Dem steht die „Trägheit des Systems“ gegenüber, die dadurch entsteht, dass Veränderungen mit einem Mehraufwand verbunden sind. Veränderungen können nur gelingen, wenn alle Betroffenen, d.h. Leistungserbringer, Patienten, Kostenträger und andere sogenannte Stakeholder, frühzeitig eingebunden sind und mittel- bis langfristig von der Veränderung profitieren. Hier spielen Motivationsarbeit und Schulungen und nicht zuletzt auch finanzielle Aufwandsentschädigungen eine erhebliche Rolle.

Interessenkonflikt: Die Autoren geben an, dass kein Interessenkonflikt besteht.

\section{Institute}

${ }^{1}$ Zentrum für Medizinische Versorgungsforschung, Psychiatrische und Psychotherapeutische Klinik, Friedrich-Alexander-Universität ErlangenNürnberg, Erlangen

${ }^{2}$ Bayerisches Landesamt für Gesundheit und Lebensmittelsicherheit (LGL) Nürnberg, Nürnberg

${ }^{3}$ Institut und Poliklinik für Arbeits-, Sozial- und Umweltmedizin der

Friedrich-Alexander-Universität Erlangen-Nürnberg, Erlangen

${ }^{4}$ Klinik für Psychiatrie und Psychotherapie, Universitätsklinik Erlangen,

Friedrich-Alexander-Universität Erlangen-Nürnberg, Erlangen

${ }^{5}$ Klinik für Psychiatrie und Psychotherapie, Rheinische Friedrich-WilhelmsUniversität Bonn und Medizinischer Dienst der Krankenversicherung in Bayern, München

${ }^{6}$ Interdisziplinäres Zentrum für Health Technology Assessment (HTA) und Public Health, Friedrich-Alexander Universität Erlangen-Nürnberg, Nationales BMBF-Spitzencluster ,Exzellenzzentrum für Medizintechnik Medical Valley EMN`, Erlangen

\section{Literatur}

1 Lohr KN, Steinwachs DM. Health services research: an evolving definition of the field. Health Serv Res 2002; 37: 7-9

2 Phillips $C D$. What do you do for a living? Toward a more succinct definition of health services research. BMC. Health Serv Res 2006; 6: 117

3 Schrappe M, Glaeske G, Gottwik M et al. Memorandum II zur Versorgungsforschung in Deutschland: „Konzeptionelle, methodische und strukturelle Voraussetzungen der Versorgungsforschung“. Deutsche Medizinische Wochenschrift 2005; 130: 2918-2922

4 Pfaff H, Schrappe M. Einführung in die Versorgungsforschung. In: Pfaff $\mathrm{H}$, Neugebauer EAM, Glaeske G et al., Hrsg. Lehrbuch Versorgungsforschung: Systematik-Methodik-Anwendung. Stuttgart: Schattauer; 2011

5 Bundesärztekammer. Definition und Abgrenzung der Versorgungsforschung. Berlin: Arbeitskreis „Versorgungsforschung“ beim Wissenschaftlichen Beirat; 2004

6 Lauterbach K, Schrappe M. Gesundheitsökonomie, Qualitätsmanagement und Evidence-based Medicine. Stuttgart: Schattauer; 2004
7 Bundesärztekammer. Definition der Versorgnungsforschung. 2006; Im Internet http://www.bundesaerztekammer.de/page.asp?his=6.3289. 3293.3294 Zugriff: 2013/11/13

8 Pfaff $H$. Versorgungsforschung - Begriffsbestimmung, Gegenstand und Aufgaben. In: Pfaff H, Schrappe M, Lauterbach KW et al., Hrsg. Gesundheitsversorgung und Disease Management: Grundlagen und Anwendungen der Versorgungsforschung. Bern: Verlag Hans Huber; 2003; 13-23

9 Perleth M, Busse R. Health Technology Assessment (HTA) - Teil und Methode der Versorgungsforschung. Gesundh ökon Qual manag 2004; 9: $172-176$

10 Clancy C, Eisenberg J. Outcomes Research: Measuring the End Results of HealthCare. Science 1998; 282: 245-246

11 WHO. ICF - The International Classification of Functioning, Disability and Health. Genf: World Health Organization; 2001

12 Porzsolt F, Geier J. Aufgaben der Versorgungsforschung aus Sicht der Klinischen Ökonomik. Monitor Versorgungsforschung 2013; 32-35

13 Müller H, Schaefer C. Surrogatparameter und patientenrelevante Endpunkte: Trugschlüsse und Konsequenzen. Monitor Versorgungsforschung 2013; 5-10

14 Mühlbacher AC, Bethge S, Junhnke C. „Patientenrelevante Endpunkte“ Bewertung aus der Perspektive der Patienten? Monitor Versorgungsforschung 2013; 11-14

15 BMG. Pressemitteilung Nr. 58: Eine flächendeckende bedarfsgerechte medizinische Versorgung bleibt auch in Zukunft in ganz Deutschland gesichert. In: Pressestelle ed.Berlin: Bundesministerium für Gesundheit; 01.12.2011

16 Neugebauer EAM, Icks A, Schrappe M. Memorandum III: Methoden für die Versorgungsforschung (Teil 2). Gesundheitswesen 2010; 72: 739-748

17 Pfaff H, Glaeske G, Neugebauer EAM et al. Memorandum III: Methoden für die Versorgungsforschung (Teil 1). Gesundheitswesen 2009; 71: 505-510

18 Glaeske G, Augustin M, Abholz $H$ et al. Epidemiologische Methoden für die Versorgungsforschung. Gesundheitswesen 2009; 71: 685-693

19 DNVF. Eckpunkte eines „Nationalen Aktionsplans für Versorgungs forschung“. 2013; Im Internet http://www.netzwerk-versorgungsfor schung.de/uploads/DKVF\%202013/Erklärung \%20-\%20Nationaler\%20 Aktionsplan\%20für\%20Versorgungsforschung.pdf Zugriff: 07.01.2014

20 Rainer M, Jungwirth S, Krüger-Rainer C et al. Pflegende Angehörige von Demenzerkrankten: Belastungsfaktoren und deren Auswirkung. Psychiatr Prax 2002; 29: 142-147

21 Perren S, Schmid R, Wettstein A. Caregivers' adaptation to change: the impact of increasing impairment of persons suffering from dementia on their caregivers' subjective well-being. Aging Ment Health 2006; 10: $539-548$

22 Gräßel E, Adabbo R. Perceived burden of informal caregivers of a cronically ill older family member. GeroPsych 2011; 24: 143-154

23 Eichenseer B, Graessel E., Hrsg. Aktivierungstherapie für Menschen mit Demenz - motorisch - alltagspraktisch - kognitiv - spirituell. 1. Aufl.München: Elsevier; 2011

24 Graessel E, Viegas $R$, Stemmer $R$ et al. The Erlangen Test of Activities of Daily Living: first results on reliability and validity of a short performance test to measure fundamental activities of daily living in dementia patients. Int Psychogeriatr 2009; 21: 103-112

25 Luttenberger K, Schmiedeberg A, Grassel E. Activities of daily living in dementia: revalidation of the E-ADL test and suggestions for further development. BMC Psychiatry 2012; 12: 208

26 Graessel E, Stemmer R, Eichenseer B et al. Non-pharmacological, multicomponent group therapy in patients with degenerative dementia: a 12-month randomised, controlled trial. BMC Med 2011; 9: 129

27 Luttenberger K, Hofner B, Graessel E. Are the effects of a non-drug multimodal activation therapy of dementia sustainable? follow-up study 10 months after completion of a randomised controlled trial. BMC Neurol 2012; 12: 151

28 Rothman M, Beltran P, Cappelleri J et al. Patient-Reported Outcomes: Conceptual Issues. Value Health 2007; 10: 66-75

29 FDA. Patient Reported Outcome Measures: Use in Medical Product Development to Support Labeling Claims. 2009; Im Internet http:// www.fda.gov/downloads/Drugs/Guidances/UCM193282.pdf Zugriff: 07.01.2014

30 Menn P, Holle R, Kunz S et al. Dementia care in the general practice setting: a cluster randomized trial on the effectiveness and cost impact of three management strategies. Value Health 2012; 15: 851-859

31 Neugebauer EAM. Von der Stammzelle zur Lebensqualität - ein integratives Forschungskonzept in der operativen Medizin. Deutsche Gesellschaft für Chirurgie Mitteilungen 2006; 2/2006: 100-105 